\title{
Evaluation of Phlebotomy-Related Anxiety, Pain and Safety in A Mexican General Hospital Using Winged ⿶ำ Blood Collection Sets
}

\author{
Andrea Flores Ibarra*1 and Silvia Villanueva ${ }^{2}$ \\ ${ }^{1}$ Institute of Chemistry, Mexico \\ ${ }^{2}$ Hospital General, Mexico
}

Received: 非 January 19, 2019; Published: 制January 29, 2019

*Corresponding author: Andrea Flores Ibarra, Institute of Chemistry, Mexico

\begin{abstract}
Introduction: The medicinal laboratory workflow heavily depends on phlebotomy, in order to obtain a representative sample from the patients to perform blood analysis with diagnostic value. Patient satisfaction is related with the treatment received from the hospital personnel, more specifically from the phlebotomist, as well as with not producing a hematoma after the punction and that the sample is obtained in the first attempt. Meanwhile, the phlebotomist encounters their own series of complications, ranging from safety issues that may result from self-punctioning to the necessity of preserving sample quality.
\end{abstract}

Objectives: The objective of this study was to evaluate the rates of pain and anxiety in recurring patients as well as the phlebotomists' safety and rate of success to improve patient care at the Hospital General 'Dr. Manuel Gea González' in Mexico City.

Methodology: We conducted a study with 2260 recurring patients in the Hospital General 'Dr. Manuel Gea González' to compare the rate of pain and anxiety in patients as well as the phlebotomists safety and rate of success during phlebotomy comparing conventional blood collection needles with BD Vacutainer® Winged Blood Collection Set devices UltraTouch and Push Button by conducting surveys after each phlebotomy.

Results: $99.1 \%$ of patients experienced less pain and anxiety during phlebotomy and $100 \%$ of the times the phlebotomists were succesful in collecting blood with one try as well as protecting themselves from accidental self-punctioning when using one of the BD Vacutainer ${ }^{\circledR}$ Winged Blood Collection Set devices UltraTouch and Push Button.

Conclusion: As new technologies become readily available for hospitals, we conclude that switching to new blood collection devices will improve significantly all the aforementioned complications during phlebotomy.

\section{Introduction}

A The medicinal laboratory workflow heavily depends on phlebotomy, rendering it a crucial step in which a blood sample has to be obtained with efficiency and in the best conditions in order to obtain a representative sample from the patients to perform blood analysis. The new technologies and quality indices in laboratories set both in hospitals or as points-of-care, show clear and promising tendencies to improve and personalize sample collection for each patient. Specifically, venous blood collection is, if not the most frequently requested, one of the most important samples needed to perform medicinal laboratory analysis [1]. Every phlebotomy has certain quality requirements that must be complied with as the minimum necessary to ensure the two main purposes in a medicinal laboratory: patient satisfaction and results with diagnostic value [2]. As new technologies become available, the laboratories see the need to keep improving their patients' experience as well as ensuring sample quality, always aiming to meet intra- and interindividual variability and personalize each phlebotomy and test [1].

Being the first-contact person to the patient in a laboratory, the phlebotomist plays one of the most important roles in customer service. Interpersonal as much as technical abilities are key to perform their job [3]. The College of American Pathologists conducted a satisfaction analysis among patients of 393 institutions and reported that this satisfaction is related, in first place, with the treatment received by the phlebotomist, and in second and third place, with the absence of hematomas and a successful blood 
collection occurring in the first attempt [4]. More importantly, many more serious complications may arise during phlebotomy. In 2008, the International Society of Blood Transfusion classified the damages a patient can experience during phlebotomy in three categories: damage to a never by a hematoma, direct damage to a nerve or tendon, and acute generalized pain in the arm. Additionally, the phlebotomist encounters their own series of complications. Regarding this, the American Nursing Association and the World Health Organization have reported that over $80 \%$ of hypodermic needle wounds in phlebotomists caused by accidental self-punctures can be avoided using safer devices [5-10]. While self functioning is particularly concerning when the patient presents a blood-borne disease, other complications are also important, such as internal bleeding, fainting, localized swelling and thrombophlebitis. Finally, once a blood sample has been obtained without any complications to the patient or the phlebotomist, this sample may not have analytical quality and be rejected by the laboratory because it is hemolyzed [11-16].

\section{Study Objectives}

The aforementioned complications during and after phlebotomy have led us to set the objective of this study to evaluate the rates of pain and anxiety in recurring patients as well as the phlebotomists' safety and rate of success in obtaining analytical quality samples to improve patient care at the Hospital General 'Dr. Manuel Gea González' in Mexico City.

\section{Methods and Patients}

A total of 2260 patients were randomly selected among all recurring patients at the Hospital General 'Dr. Manuel Gea González' in a time span of eight months and their written consent was obtained for each comparation during phlebotomy. Every phlebotomy conducted was performed twice in each patient, with a conventional blood collection needle in one arm and with a winged blood collection set randomizing between BD Vacutainer ${ }^{\circledR}$
UltraTouch or Push Button. After finalizing the blood collection, a satisfaction survey (Table 1) was filled in the following fashion: the patients answered one question regarding the level of pain and anxiety they experienced during phlebotomy and the phlebotomists answered two questions regarding safety as the likelihood of selfpunctioning and rate of success as being able to obtain and adequate blood sample for the studies in the first attempt and the quality of the sample from visible hemolysis.

Table 1: Satisfaction survey.

\section{Satisfaction Survey \\ Patient: Did you experience more or less pain and anxiety with the winged blood collection set than with a conventional needle? \\ Phlebotomist: Do you feel safer regarding accidental self-punctioning with the winged blood collection set over a conventional needle? \\ Phlebotomist: Was it easier to obtain a blood sample in the first attempt with no visible hemolysis using a winged blood collection set over a conventional needle?}

\section{Results}

The questions in the survey were answered immediately after each phlebotomy. These questions are presented in (1) as they were to the patients and phlebotomists. The total of patients that answered positively to the question regarding pain and anxiety was 2240 , with 20 negative answers. Regarding the confidence the winged blood collection set provides to phlebotomists in terms of self-punctioning likelihood and obtention of analytical quality samples, all answers were positive. The needle retraction in the BD Vacutainer $®$ winged blood collection sets used for the tests is of 0.7 seconds and the security device should be triggered while the needle is still inside the patient [12], making any blood-borne disease transmission to the phlebotomist completely unlikely. The needle technology [12] also gave a positive response regarding sample hemolysis (Figure 1).



Figure 1. 


\section{Discussion}

Patient satisfaction is related to several factors that must be controlled by the medicinal laboratory in order to comply with the quality requirements of the healthcare area. This may be measured by indices such as the number of attempts needed before obtaining a blood sample, and once obtained, that this sample has the analytical quality to have results with diagnostic value. Additionally, it is fundamental that the phlebotomists perform their jobs with confidence against blood-borne diseases. Taking this into account, the Hospital General "Dr. Manuel Gea González" validated the use of winged blood collection sets compared with conventional needles for phlebotomy to evaluate the following parameters. For the patients, the rate of pain and anxiety patients experience with one device or the other. For the phlebotomists, with all the evaluations positive towards the winged sets, the amount of punctions needed to obtain the blood sample, the safety of the device against selfpunction, and the sample quality measured as visible hemolysis. While hemolysis is an objective index to evaluate phlebotomy, as well as obtaining an adequate blood sample in the first attempt, pain and anxiety are more subjective and depend on patient sensitivity or even in their particular mood at the precise moment of the puncture. We attributed to this that not all the answers to the first question were positive, although a vast majority of them were, meaning that both the pain and anxiety sensations were reduced in patients with the use of the winged blood collection sets.

\section{Conclusion}

The results clearly show that the utilization of the winged blood collection sets used in this study will improve both the services of phlebotomy and the diagnostic value of the clinical laboratory analysis offered in the Hospital General "Dr. Manuel Gea González". By reducing the number of phlebotomy attempts to collect an analytical quality blood sample to only one, by reducing the phlebotomist concern in acquiring a blood-borne disease, and by reducing the frequency of hemolysis and thus the need to repeat tests, the quality requirements for the medicinal laboratory can be met in a way in which the patients feel safe to come back to this hospital when seeking a healthcare facility. This is the first study of this kind performed in Mexico with the winged blood collection sets, and its rate of success allows us to recommend their use in the medicinal laboratory. Further studies will be conducted in which hemolysis is assessed quantitatively by means of measuring potassium and lactate dehydrogenase.

\section{ISSN: 2574-1241}

\section{DOI: $10.26717 / B J S T R .2019 .13 .002455$}

Andrea Flores Ibarra. Biomed J Sci \& Tech Res

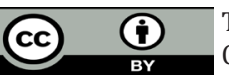

This work is licensed under Creative Commons Attribution 4.0 License

Submission Link: https://biomedres.us/submit-manuscript.php

\section{References}

1. (2012) American Association of Blood Banks. Biovigilance.

2. (2000) Nurses win protection under new OSHA regulations. American Journal of Nursing 100(2): 20.

3. Brott TG, Halperin JL, Abbara S, J Michael Bacharach, John D Barr, et al. (2011) Guideline on the management of patients with extracranial carotid and vertebral artery disease. Journal of the American College of Cardiology 57(8): 17-94.

4. Dale JC, Howanitz PJ (2015) Patient satisfaction in phlebotomy: a College of American Pathologists' Q-probes study. Laboratory medicine 27(3): 188-192.

5. Centers for Disease Control and Prevention.

6. Dahlquist LM, Busby SM, Slifer KJ, Tucker CL, Eischen S, et al. (2002) Distraction for children of different ages who undergo repeated needlesticks. Journal of Pediatric Oncology Nursing 19(1): 22-34.

7. Goldman M, Land K, Robillard P, Wiersum Osselton J (2015) Development of standard definitions for surveillance of complications related to blood donation. Vox Sanguinis. Short report 110(2): 185-188.

8. Katz L, Benjamin RJ, Tomasulo PA (2014) Interventions for vasovagl reactions at Alliance of Blood Operator blood establishments: an international survey for the ABO medical working group. Transfusion 14: 54-110A.

9. Kennedy RM, Luhmann J, Zempsky WT (2008) Clinical implications of unmanaged needle insertion pain and distress in children. Pediatrics 122(3): S130-S132.

10. Lin JC, Strauss RG, Kulhavy, Johnson KJ, Zimmerman MB, et al. (2000) Phlebotomy overdraw in the neonatal intensive care nursery. Pediatrics 106(2): 19.

11. Lippi G, Blanckaert N, Bonini P, Green S, Kitchen S, et al. (2008) Haemolysis: an overview of the leading cause of unsuitable specimens in clinical laboratories. Clinical Chemistry and Laboratory Medicine 46(6): 764-772.

12. Mouser A, Uettwiller Geiger D, Plokhoy E, Julie Berube, Aparna Jha Ahuja, et al. (2017) Evaluation of pain and specimen quality by use of a novel 25-gauge blood collection set with ultra-thin wall cannula ad 5-bevel tip design. Journal of Applied Laboratory Medicine 2(2): 201-210.

13. (2012) World Health Organization. How to reduce HIV/AIDS effects in the nursing and obstetrics personnel. American Nursing Association.

14. Sanavio B, Krol S (2015) On the slow diffusion of point-of-care systems in therapeutic drug monitoring. Frontiers in Bioengineering and Biotechnology. Review 3(20): 1-15.

15. Wayne PA (2017) Clinical and Laboratory Standards Institute. Collection of diagnostic venous blood specimens. In Wayne PA(Eds.) ( $7^{\text {th }}$ Edn.) CLSI standard GP42.

16. Lippi G, von Meyer A, Cadamuro J, Simundic AM (2018) Blood Sample Quality. Diagnosis.

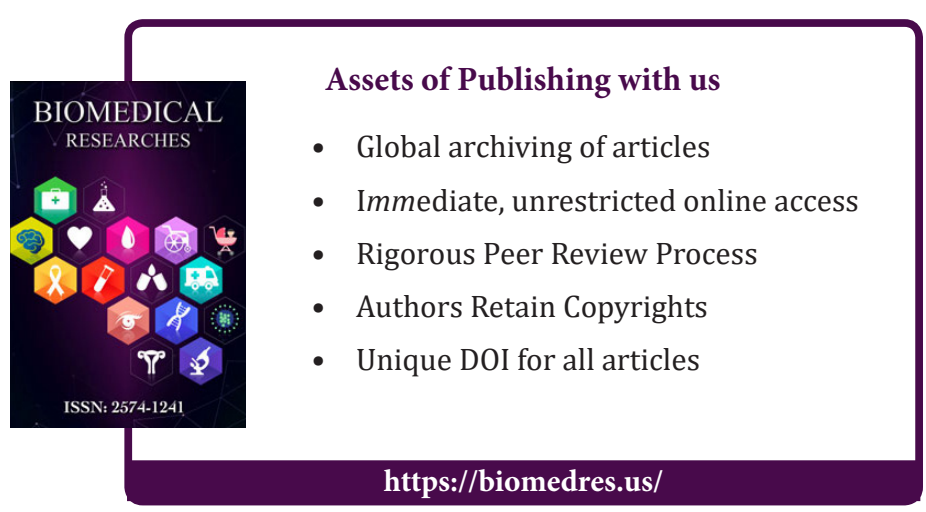

Cite this article: Andrea Flores Ibarra, Silvia Villanueva. Evaluation of Phlebotomy-Related Anxiety, Pain and Safety in A Mexican General Hospital Using Winged Blood Collection Sets. Biomed J Sci \& Tech Res 13(5)-2019. BJSTR. MS.ID.002455. DOI: 10.26717/ BJSTR.2019.13.002455. 